\title{
EFEITOS DA QUALIDADE E QUANTIDADE DA ÁGUA DE IRRIGAÇÃO SOBRE O COENTRO CULTIVADO EM SUBSTRATO INICIALMENTE SALINO ${ }^{1}$
}

\author{
José Francismar de Medeiros ${ }^{2}$, Damião Santos de Medeiros ${ }^{3}$, Francisco Queiroz Porto Filho ${ }^{4}$ \\ e Iseni C. C. Nogueira ${ }^{4}$
}

\begin{abstract}
RESUMO
Com o objetivo de se estudar os efeitos da salinidade na produção de coentro cultivar aromática foram ensaiados, em canteiros adubados com esterco, três tipos de água $\left(S_{1}=0,56, S_{2}=2,41\right.$ e $S_{3}=3,93$ dS.m $\left.{ }^{-1}\right)$ e dois níveis de irrigação $\left(\mathrm{N}_{1}=1,03 \mathrm{ETo}\right.$ e $\left.\mathrm{N}_{2}=1,25 \mathrm{ETo}\right)$ em esquema fatorial $3 \times 2$, cujos resultados mostraram que a produção de folhas verdes de coentro, que está relacionada à altura, ao número de hastes por planta e ao stand final, foi afetada significativamente pela salinidade do solo resultante do esterco e dos tratamentos estudados e que a produção comercial de coentro só foi satisfatória para os tratamentos cuja salinidade do solo ficou abaixo de 5,1 dS.m ${ }^{-1}$.
\end{abstract}

Palavras-chave: Coriandrum sativum, salinidade, irrigação

\section{EFFECTS OF QUALITY AND AMOUNT OF IRRIGATION WATER ON CORIANDER CROP CULTIVATED IN INITIALLY SALINE SUBSTRATE}

\begin{abstract}
The objective of this research was to evaluate the effects of salinity and amount of irrigation water on coriander yield. The experiment was carried out in manure bed, using three different kinds of water $\left(\mathrm{S}_{1}=0.56, \mathrm{~S}_{2}=2.41\right.$, and $\left.\mathrm{S}_{3}=3.93 \mathrm{dS} . \mathrm{m}^{-1}\right)$ and two levels of irrigation $\left(\mathrm{N}_{1}=1.03 \mathrm{ETo}\right.$ and $\left.\mathrm{N}_{2}=1.25 \mathrm{ETo}\right)$ in a $3 \times 2$ factorial scheme. The results showed that the production of green leaved coriander, which is related to height, number of stems per plant and the final stand, was considerably affected by the soil salinity resulting from the manure and studied treatments. The comercial coriander production was satisfactory for the treatment where soil salinity remained below $5.1 \mathrm{dS} . \mathrm{m}^{-1}$.
\end{abstract}

Key words: Coriandrum sativum, salinity, irrigation

\section{INTRODUÇÃO}

O coentro (Coriandrum sativum) é uma espécie olerícola consumida em todas as regiões do Brasil, principalmente na região Nordeste. Segundo Filgueira (1982) as folhas, com sabor e aroma muito ativos e peculiares, são incorporadas às receitas culinárias de peixes e às sopas portuguesas e em diversos pratos típicos do Nordeste do Brasil. Souza (1981) afirma que o coentro se situou, no ano de 1977 , em $10^{\circ}$ lugar e, em 1978 , atingiu o $5^{\circ}$ lugar entre os produtos comercializados na CEASA, PE, sendo

\footnotetext{
${ }^{1}$ Parte do trabalho de monografia do segundo autor

2 Eng. Agr., Doutor, Depto. Eng. Agrícola, ESAM; CP 137 - CEP 59625-900, Mossoró, RN

${ }^{3}$ Eng. Agr., Projetos Alternativos, Caicó, RN

${ }^{4}$ Prof. Adj., M.Sc., Depto. Eng. Agrícola e Fitotecnia, respectivamente, ESAM, Mossoró, RN
} 
ultrapassado pelo tomate, pela cebola, batatinha e abóbora. Matias (1986) ressalta que, no Rio Grande do Norte, a quantidade média de coentro comercializado na CEASA em 1985 atingiu $23.900 \mathrm{~kg}$.

Mesmo sendo uma cultura de destaque comercial, têm sido poucos os estudos que visam melhorar as técnicas de produção dessa olerícola (Souza, 1981; Filgueira, 1993).

Em virtude das condições climáticas da região, o coentro sempre é cultivado com o uso da irrigação; geralmente, é uma cultura explorada em pequenas áreas, sendo utilizada água proveniente de pequenas fontes (açudes pequenos, poços amazonas, poços naturais em leitos de rios, poços tubulares etc.). Essa água, dependendo da época do ano, geralmente contém níveis elevados de sais (Leprun, 1983; Medeiros, 1992), e no caso de ser usada na irrigação de algumas culturas, pode prejudicar o seu desenvolvimento, diminuindo conseqüentemente a produção.

Quando se utiliza água de irrigação com concentração elevada de sais, é necessário se conhecer a salinidade acima da qual a planta começa a ser afetada. Segundo Maas \& Hoffman (1977) as hortaliças, de maneira geral, são as culturas mais sensíveis à salinidade.

Medeiros et al. (1993b) estudando o efeito de diferentes níveis de salinidade da água de irrigação $(0,55,1,23,2,27,3,41$ e 4,50 dS. $\mathrm{m}^{-1}$ ) e uma fração de lixiviação de 0,10 , sobre duas cultivares de coentro, concluíram que os níveis de salinidade afetaram os vários parâmetros estudados, como peso fresco e altura de plantas, por ocasião da colheita de folhas verdes, produção de frutos e seus componentes de produção. A diminuição nos rendimento de folhas verdes e de frutos para o nível mais alto de salinidade, em relação à produção obtida para a água de baixa salinidade, foi de $33 \%$ e $29 \%$, respectivamente.

Filgueira (1993) estudando diferentes níveis de salinidade do solo $\left(5,6,6,6,7,6,9,1\right.$ e 11,6 dS.m $\left.{ }^{-1}\right)$ no comportamento de duas cultivares de coentro, observou que a cultivar Português foi mais afetada pela salinidade que a Palmeira, pois esta última, além de retardar consideravelmente a colheita de folhas verdes, não produziu frutos durante o ciclo normal da cultura.

Este trabalho teve como objetivo estudar a influência da qualidade e da quantidade da água de irrigação no rendimento do coentro para a produção de folhas verdes, cultivado em canteiros com substrato inicialmente salino, composto de solo e esterco de curral.

\section{MATERIAIS E MÉTODO}

O experimento foi conduzido em Mossoró, $\mathrm{RN}\left(5^{\circ} 11^{\text {' }}\right.$ de latitude Sul e $37^{\circ} 20^{\prime}$ de longitude Oeste) na horta da Escola Superior de Agricultura de Mossoró (ESAM) no período de setembro a novembro de 1993, em solo classificado como Podzólico Vermelho-Amarelo Equivalente Eutrófico, segundo classificação apresentada pelo Ministério da Agricultura (BRASIL, 1971). O substrato usado nos canteiros constituiu-se de uma mistura deste solo com esterco $\left(8 \mathrm{~kg} \cdot \mathrm{m}^{-2}\right)$ cujas características físicas e químicas encontram-se na Tabela 1.

O comportamento das condições climáticas durante a realização do experimento está apresentado na Tabela 2 ,sendo que não se verificou precipitação pluviométrica.

Os tratamentos consistiram da combinação de dois fatores:
Tabela 1. Características físico-químicas do substrato dos canteiros, constituídos de solo e esterco, na profundidade de 0 a $20 \mathrm{~cm}$, por ocasião da semeadura

\begin{tabular}{|c|c|c|c|}
\hline Descrição & & Unidade & Valor \\
\hline CEes* & & $\mathrm{dS} . \mathrm{m}^{-1}$ & 5,0 \\
\hline $\mathrm{PH}\left(\mathrm{H}_{2} \mathrm{O}\right)$ & & - & 7,3 \\
\hline $\mathrm{Ca}$ & & $\mathrm{cmol}_{\mathrm{c}} \cdot \mathrm{kg}^{-1}$ & 11,7 \\
\hline $\mathrm{Mg}$ & & $\mathrm{cmol}_{\mathrm{c}} \cdot \mathrm{kg}^{-1}$ & 5,8 \\
\hline $\mathrm{K}$ & & $\mathrm{cmol}_{\mathrm{c}} \cdot \mathrm{kg}^{-1}$ & 3,5 \\
\hline $\mathrm{Na}$ & & $\mathrm{cmol}_{\mathrm{c}} \cdot \mathrm{kg}^{-1}$ & 1,8 \\
\hline $\mathrm{Al}$ & & $\mathrm{cmol}_{\mathrm{c}} \cdot \mathrm{kg}^{-1}$ & 0,0 \\
\hline $\mathrm{P}$ & & $\mathrm{cmol}_{\mathrm{c}} \cdot \mathrm{kg}^{-1}$ & 2,2 \\
\hline Densidade do solo & & g. $\mathrm{cm}^{-3}$ & 1,09 \\
\hline \multicolumn{4}{|l|}{ Umidade do solo } \\
\hline & $\begin{array}{r}0 \mathrm{kPa} \\
10 \mathrm{kPa}\end{array}$ & $\begin{array}{l}\mathrm{cm}^{3} \cdot \mathrm{cm}^{-3} \\
\mathrm{~cm}^{3} \cdot \mathrm{cm}^{-3}\end{array}$ & $\begin{array}{l}0,546 \\
0,286\end{array}$ \\
\hline & $30 \mathrm{kPa}$ & $\mathrm{cm}^{3} \cdot \mathrm{cm}^{-3}$ & 0,264 \\
\hline
\end{tabular}

Tabela 2. Dados climáticos ${ }^{1}$ médios por período, durante o desenvolvimento da pesquisa

\begin{tabular}{lccccc}
\hline & \multicolumn{5}{c}{ Períodos $^{2}$} \\
\cline { 2 - 6 } Discriminação & $(1)$ & $(2)$ & $(3)$ & $(4)$ & $(5)$ \\
\hline Temp. média $\left({ }^{\circ} \mathrm{C}\right)$ & 28,3 & 28,2 & 29,0 & 29,3 & 29,3 \\
Vel. vento $\left(\mathrm{m} . \mathrm{s}^{-1}\right)$ & 4,9 & 4,9 & 4,9 & 5,2 & 5,0 \\
U.R. $(\%)$ & 56,0 & 62,0 & 56,0 & 55,0 & 54,0 \\
ECA $^{3}\left(\mathrm{~mm} . \mathrm{dia}^{-1}\right)$ & 10,2 & 8,4 & 10,0 & 10,7 & 11,1 \\
$\mathrm{ETo}^{4}\left(\mathrm{~mm} \cdot \mathrm{dia}^{-1}\right)$ & 6,7 & 5,4 & 6,4 & 6,9 & 7,0 \\
\hline
\end{tabular}

${ }^{1}$ Dados obtidos junto à Estação Meteorológica no Campus da ESAM - Mossoró, RN

2 (1), (2), (3), (4) e (5) correspondem, respectivamente, aos períodos de 16-20/09, 21/09-06/10, 07-21/10, 22/10-04/11 e 05-16/11

${ }^{3}$ Evaporação do tanque classe $\mathrm{A}$

${ }^{4}$ Evapotranspiração de referência, estimada diariamente, segundo Doorenbos \& Pruit (1977) a partir da ECA

tipos de água e níveis de irrigação. Foram utilizados três tipos de água com salinidades diferentes $\left(S_{1}, S_{2}\right.$ e $\left.S_{3}\right)$; a água de salinidade $S_{1}$ procedeu de poço artesiano, localizado no Campus da ESAM, com CE de 0,56 dS.m ${ }^{-1}$; a de salinidade $S_{3}$ procedeu de poço escavado no aqüífero calcário, instalado vizinho à horta da ESAM, com CE de 3,93 dS.m ${ }^{-1}$ e a de salinidade $\mathrm{S}_{2}$ correspondeu à mistura eqüitativa das águas provenientes dos dois poços, ficando com uma $\mathrm{CE}$ de $2,41 \mathrm{dS} / \mathrm{m}$. Os níveis da quantidade de água de irrigação aplicada foram dois: $\mathrm{N}_{1}$, que correspondeu à água necessária para manter a tensão de água no solo a $5 \mathrm{kPa}$, e $\mathrm{N}_{2}$, que correspondeu à quantidade de $\mathrm{N}_{1}$ acrescida de $20 \%$, correspondendo, em média, respectivamente, a 1,03 e 1,25 vezes a evapotranspiração de referência (ETo) verificada durante o cultivo do coentro. Estas lâminas de irrigação deverão, certamente, ter produzido frações de lixiviação superiores a 20\%, pois de acordo com Doorenbos \& Pruit (1977) o coeficiente de cultivo médio até o florescimento para as olerícolas fica abaixo de 0,8 .

O delineamento experimental foi o inteiramente casualizado, no esquema fatorial $2 \times 3$ e com 4 repetições.

$\mathrm{O}$ experimento foi executado em três canteiros de $10 \mathrm{~m}$ de comprimento por $1,2 \mathrm{~m}$ de largura. Cada canteiro foi dividido em oito parcelas de $1,2 \mathrm{~m}$ de comprimento, perfazendo o total de 24 parcelas; as divisões foram feitas com lona plástica, inseridas transversalmente nos canteiros até a profundidade de $35 \mathrm{~cm}$; depois, procedeu-se, na seqüência, às seguintes práticas: irrigação para lavagem do excesso de sais proveniente do esterco incorporado, durante 21 dias, sendo $138 \mathrm{~mm}$ com água de 
salinidade $S_{1}$ durante os primeiros 5 dias para todas as parcelas e, nos dias restantes, foram aplicados $172 \mathrm{~mm}$ para $\mathrm{N}_{1}$ e $270 \mathrm{~mm}$ para $\mathrm{N}_{2}$, usando-se as águas dos respectivos tratamentos; desinfecção com formalina (formol a 2,5\%) e cobertura pelo período de 48 horas.

A semeadura foi feita manualmente, em sulcos com fileiras contínuas espaçadas $20 \mathrm{~cm}$, perfazendo 6 fileiras por parcela, utilizando-se a cultivar Aromática; não se realizaram desbastes de plantas e as capinas foram realizadas manualmente, quando necessário.

As irrigações foram efetuadas uma ou duas vezes ao dia e controladas através de tensiômetros instalados em duas parcelas do tratamento $S_{1} N_{1}$, nas profundidades de 7,5 e $22,5 \mathrm{~cm}$, de modo a manter o solo sempre próximo à capacidade de campo $(5 \mathrm{kPa})$; além disso, foram efetuadas 3 medições de umidade do solo gravimetricamente em todas as parcelas, durante o cultivo, com o objetivo de se monitorar a umidade do solo também nas demais parcelas. Com relação à lâmina aplicada por dia, a mesma foi estabelecida em função da tensão da água no solo medida nas parcelas do tratamento $\mathrm{S}_{1} \mathrm{~N}_{1}$, da condição do tempo e das observações visuais (como os produtores de coentro manejam a irrigação). O método de irrigação utilizado foi o de aspersão manual (regador).

Determinou-se, por ocasião da colheita de folhas verdes, 40 dias após a semeadura, a altura média de dez plantas de cada parcela, medida do nível do solo até a extremidade mais alta das plantas, também foram retiradas, ao acaso, dez plantas por parcela para determinação do número de hastes por planta e, para se quantificar o índice de falhas no stand final, estabeleceram-se valores que variaram de 0 a 5 , onde " 0 " foi atribuído para parcelas sem falhas, "1" para parcelas com 1 a $10 \%$ de falhas, " 2 " correspondeu às parcelas com 11 a $20 \%$ de falhas, " 3 " com 21 a $40 \%$ de falhas, " 4 " a 41 a $70 \%$ de falhas e " 5 " a mais de $70 \%$ de falhas.

A produção de folhas verdes de coentro foi expressa em kg.ha-1 por hectare, a partir da colheita das plantas por inteiro presentes na área útil da parcela, que foi de $0,48 \mathrm{~m}^{2}$.

A avaliação da salinidade do solo foi expressa em condutividade elétrica do extrato de saturação (CEes) medida nas camadas de 0 a 10, 10 a 20 e de 20 a 30cm, em intervalos de aproximadamente 14 dias; estabeleceu-se, posteriormente, a salinidade média na zona radicular da cultura para o período de cultivo, assumindo as profundidades de 10, 20 e $30 \mathrm{~cm}$; a CEes foi estimada a partir da $\mathrm{CE}$ no extrato aquoso $\left(\mathrm{CE}_{1: 5}\right)$ (Medeiros et al., 1993a).

Foram realizadas análises de variância das variáveis altura de plantas, número de hastes, índice de falhas no stand final, produção de folhas verdes de coentro e salinidade do solo na camada de 0 a $10 \mathrm{~cm}$, enquanto a comparação de média foi realizada pelo teste de Tukey, a nível de 5\% de probabilidade, conforme Gomes (1987). Para as análises estatísticas dos dados de índice de falhas no stand final e de produção, os mesmos foram transformados, respectivamente, em $\sqrt{\mathrm{x}}$ e $\ln (\mathrm{x})$ (Ferreira, 1991).

\section{RESULTADOS E DISCUSSÃO}

Na Tabela 3 são apresentadas as médias de produção de folhas verdes de coentro, em relação à salinidade da água $(\mathrm{S})$ e aos níveis de irrigação $(\mathrm{N})$. Verifica-se que a produção de coentro foi afetada significativamente pela interação dos fatores estudados. Considerando-se o efeito da salinidade da água dentro dos níveis de aplicação, observa-se que a produção da cultura foi diminuída significativamente, tanto para $\mathrm{N}_{1}$ como para $\mathrm{N}_{2}$, a medida em que a salinidade da água de irrigação foi aumentada.

Tabela 3. Produção de folhas verdes de coentro, em kg.ha-1, obtida para diferentes salinidades da água e níveis de irrigação

\begin{tabular}{|c|c|c|c|c|}
\hline \multirow{2}{*}{$\begin{array}{l}\text { Salinidade } \\
\text { da água }\end{array}$} & \multicolumn{4}{|c|}{ Níveis de irrigação* } \\
\hline & $\mathrm{N}$ & & & \\
\hline $\mathrm{S}_{1}$ & $(13.950)$ & 9,4 a $\mathrm{A}$ & $(24.128)$ & 10,05 a $\mathrm{A}$ \\
\hline $\mathrm{S}_{2}$ & $(409)$ & 5,8 b B & $(6.387)$ & $8,7 \mathrm{~b}$ A \\
\hline $\mathrm{S}_{3}$ & $(135)$ & $4,7 \mathrm{c} \mathrm{B}$ & $(955)$ & $6,8 \mathrm{c} \mathrm{A}$ \\
\hline
\end{tabular}

C.V. $=6,9 \%$

* Valores entre parênteses representam o rendimento não transformado da cultura. Letras minúsculas diferentes nas colunas e, maiúsculas, nas linhas, mostraram diferenças significativas a $5 \%$ de probabilidade

Com respeito ao efeito do nível de irrigação dentro dos valores de salinidade da água de irrigação constata-se, na Tabela 3 , que para a água menos salina $\left(\mathrm{S}_{1}\right)$ não houve diferença significativa na produção quando se irrigou com os níveis de irrigação $\mathrm{N}_{1}$ e $\mathrm{N}_{2}$; já para as duas águas mais salinas $\left(\mathrm{S}_{2}\right.$ e $\left.\mathrm{S}_{3}\right)$ a produção foi significativamente maior para o nível de aplicação de água mais elevado $\left(\mathrm{N}_{2}\right)$; esta maior produção pode ser explicada pela maior fração de lixiviação que a maior lâmina de irrigação proporciona como, também, conforme determinação, por esta haver permitido maiores conteúdos de água no solo ao longo do tempo, reduzindo os efeitos de níveis altos de salinidade do solo sobre a planta.

A redução drástica na produtividade com o aumento da salinidade, está associada, certamente, ao conjunto de variáveis (altura de planta, número de hastes e, sobretudo, ao índice de falha no stand final) que a compõe, as quais foram diminuídas com o aumento da salinidade (Tabelas 4 e 5). Como se verifica nessas tabelas, o índice de falhas foi o parâmetro mais reduzido pela salinidade da água, apresentando valores que representam falha acima de $70 \%$ para $\mathrm{S}_{3}$; os níveis elevados de salinidade promoveram queima e morte das plantas, eliminando quase

Tabela 4. Altura e número de hastes.planta ${ }^{-1}$ por ocasião da colheita de folhas verdes

\begin{tabular}{|c|c|c|c|c|c|c|}
\hline \multirow{2}{*}{$\begin{array}{l}\text { Salinidade } \\
\text { da água }\end{array}$} & \multicolumn{3}{|c|}{ Altura (mm) } & \multicolumn{3}{|c|}{$\mathrm{N}^{\mathrm{o}}$ de hastes } \\
\hline & $\mathrm{N}_{1}$ & $\mathrm{~N}_{2}$ & Média & $\mathrm{N}_{1}$ & $\mathrm{~N}_{2}$ & Média \\
\hline $\mathrm{S}_{1}$ & 171 & 220 & $196 \mathrm{a}^{*}$ & 6,2 & 6,8 & $6,4 \mathrm{a}$ \\
\hline $\mathrm{S}_{2}$ & 66 & 118 & $92 \mathrm{~b}$ & 4,4 & 5,8 & $5,1 \mathrm{~b}$ \\
\hline $\mathrm{S}_{3}$ & 44 & 85 & $64 \mathrm{~b}$ & 4,2 & 5,3 & $4,8 \mathrm{~b}$ \\
\hline Média & $94 \mathrm{~B}$ & $141 \mathrm{~A}$ & & $4,9 \mathrm{~B}$ & $6,0 \mathrm{~A}$ & \\
\hline C.V. $(\%)$ & & 23,3 & & & 9,5 & \\
\hline
\end{tabular}
cativas a nível de $5 \%$ de probabilidade

Tabela 5. Índice de falhas no stand final por ocasião da colheita de folhas-verdes

\begin{tabular}{ccc}
\hline \multirow{2}{*}{$\begin{array}{c}\text { Salinidade da } \\
\text { Água }\end{array}$} & \multicolumn{2}{c}{ Níveis de irrigação* } \\
\cline { 2 - 2 } $\mathrm{N}_{1}$ & $\mathrm{~N}_{2}$ \\
\hline $\mathrm{S}_{1}$ & $(1,1) 1,03 \mathrm{~b} \mathrm{~A}$ & $(0,9) 0,90 \mathrm{c} \mathrm{A}$ \\
$\mathrm{S}_{2}$ & $(4,8) 2,17 \mathrm{a} \mathrm{A}$ & $(2,4) 1,53 \mathrm{~b} \mathrm{~B}$ \\
$\mathrm{~S}_{3}$ & $(5,0) 2,23 \mathrm{a} \mathrm{A}$ & $(4,1) 2,02 \mathrm{a} \mathrm{A}$ \\
\hline
\end{tabular}

C.V. $=10,4 \%$

* Valores entre parênteses representam o rendimento observado da cultura sem ser transformado. Letras diferentes minúsculas nas colunas e, maiúsculas, nas linhas, apresentaram diferenças significativas a nível de $5 \%$ de probabilidade 
completamente o stand inicial no nível de salinidade mais elevado; esta queima e morte das plantas podem estar associadas ao efeito do baixo potencial osmótico da solução do solo, sobretudo no início do cultivo, e aos íons tóxicos presentes na solução do solo ( $\mathrm{Na}$ e $\mathrm{Cl}$ ) ou na água de irrigação, aplicada por aspersão. Deve-se salientar, também, que depois da emergência, embora se tenha obtido menor stand para a água menos salina, as falhas não chegaram a $30 \%$, sendo que a mortandade das plantas acentuou-se entre o $10^{\circ}$ e $20^{\circ}$ dia após a emergência.

Considerando-se os dados de altura das plantas (Tabela 4) pode-se concluir que apenas os tratamentos $\mathrm{S}_{1} \mathrm{~N}_{1}, \mathrm{~S}_{1} \mathrm{~N}_{2}$ e $\mathrm{S}_{2} \mathrm{~N}_{2}$ produziram plantas de coentro com valor comercial, haja vista sua altura ser superior a $10 \mathrm{~cm}$.

Ressalta-se, ainda, que o efeito dos tratamentos estudados sobre as plantas é, de forma geral, indireto, pois a planta tolera a salinidade da solução do solo, que depende da qualidade da água, da fração de lixiviação aplicada e da salinidade do solo antes do cultivo (Ayers \& Westcot, 1991; Rhoades et al., 1992). Analisando-se a Tabela 6, verifica-se que houve interação entre os tratamentos estudados para a salinidade do solo (CEes) na camada 0 a $10 \mathrm{~cm}$; neste caso, para as duas águas menos salinas, o maior volume de água aplicado proporcionou salinidade do solo menor, embora acima do esperado para as condições de equilíbrio dinâmico, segundo Ayers \& Westcot (1991) enquanto para a água $S_{3}$, não houve diferença significativa entre os níveis de irrigação, o que pode ser explicado pelo fato da salinidade do solo estar no início do cultivo (Figura 1) apresentando valores similares, associada ao pequeno período de irrigação.

Tabela 6. Salinidade média do solo $\left(\mathrm{dS} . \mathrm{m}^{-1}\right)$, no período de cultivo, na camada superficial do solo de $10 \mathrm{~cm}$, obtida sob diferentes salinidade da água e níveis de irrigação

\begin{tabular}{ccc}
\hline \multirow{2}{*}{$\begin{array}{c}\text { Salinidade da } \\
\text { água }\end{array}$} & \multicolumn{2}{c}{ Níveis de irrigação* } \\
\cline { 2 - 3 } $\mathrm{N}_{1}$ & $\frac{\mathrm{N}_{2}}{3,48 \mathrm{c} \mathrm{B}}$ \\
$\mathrm{S}_{1}$ & $4,79 \mathrm{c} \mathrm{A}$ & $5,06 \mathrm{~b} \mathrm{~B}$ \\
$\mathrm{~S}_{2}$ & $6,60 \mathrm{~b} \mathrm{~A}$ & $8,44 \mathrm{a} \mathrm{A}$ \\
$\mathrm{S}_{3}$ & $7,99 \mathrm{a} \mathrm{A}$ & 8 \\
\hline
\end{tabular}

C.V. $=9,5 \%$

* Letras minúsculas comparam médias nas colunas e, maiúsculas, nas linhas

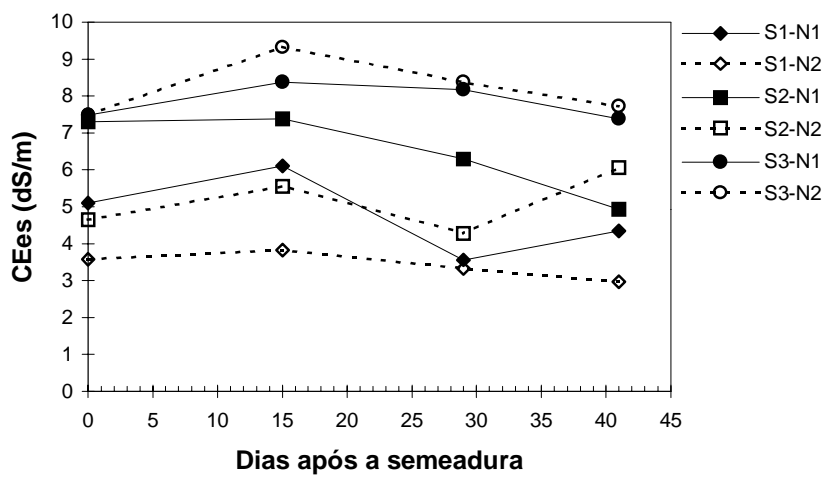

Figura 1. Salinidade do solo ao longo do ciclo da cultura, na camada de 0 a $10 \mathrm{~cm}$ de profundidade para cada tratamento

A análise da salinidade do solo apenas para camada superficial foi devida ao fato da salinidade média do solo, considerando-se a camada 0 a $30 \mathrm{~cm}$, não ter correlação significativa, sendo bem maior $\left(r=0,76^{* *}\right)$ para a camada superficial e, também, pelo fato do sistema radicular ter ficado apenas na camada superficial.
Ainda se observa, na Figura 1 que, de modo geral, a salinidade do solo apresentou tendência de crescimento nos primeiros 15 dias, seguida de redução leve, embora a análise de variância tenha demonstrado que não houve efeito significativo a 0,05 de probabilidade para período de amostragem; esperavase, portanto, diminuição da salinidade inicial do solo, pois a mesma era relativamente elevada, sobretudo para as águas $\mathrm{S}_{1} \mathrm{e}$ $\mathrm{S}_{2}$ e, ainda, devido às frações de lixiviação relativamente altas adotadas; é importante se constatar, também, que os tratamentos que produziram plantas de coentro comerciais $\left(\mathrm{S}_{1} \mathrm{~N}_{1}, \mathrm{~S}_{1} \mathrm{~N}_{2}\right.$ e $\mathrm{S}_{2} \mathrm{~N}_{2}$ ) tinham a salinidade do solo, por ocasião da semeadura, abaixo de 5,0 dS.m ${ }^{-1}$ e que, ao longo do ciclo, nunca superou 6,0 ; no entanto, nos outros tratamentos, que praticamente não produziram, a salinidade do solo até o $20^{\circ}$ dia sempre esteve acima de 7,0 dS.m ${ }^{-1}$. Segundo Maas \& Hoffmam (1977) a maioria das plantas é mais sensível à salinidade no início do ciclo, o que pode trazer conseqüências negativas para o desenvolvimento completo da cultura. Filgueira (1993) verificou que as cultivares de coentro Palmeira e Português não apresentaram produção comercial para salinidade do solo acima de 7,6 dS.m ${ }^{-1}$ e, conjuntamente com os dados obtidos por Medeiros et al. (1993b) pode classificar-se o coentro como sendo uma cultura sensível a moderadamente sensível à salinidade.

$\mathrm{Na}$ Figura 2 estão os dados do rendimento de folhas verdes de coentro em relação à salinidade média do solo, resultante da aplicação dos diferentes tratamentos estudados. Verifica-se redução no rendimento da cultura em função da salinidade do solo na camada superficial, exceto para o tratamento $\mathrm{S}_{3} \mathrm{~N}_{2}$. Revendo-se as Tabelas 3 e 6, verifica-se que, embora não tenha havido diferença significativa para salinidade do solo entre os tratamentos $\mathrm{S}_{3} \mathrm{~N}_{1}$ e $\mathrm{S}_{3} \mathrm{~N}_{2}$, a produção obtida no tratamento $\mathrm{S}_{3} \mathrm{~N}_{2}$ foi significativamente maior que em $\mathrm{S}_{3} \mathrm{~N}_{1}$; isto pode ter ocorrido devido ao maior nível de umidade do solo integrado no tempo resultante do maior nível de aplicação de água, o que reduz o efeito da salinidade do solo para o desenvolvimento das plantas, pois mantém os sais do solo mais diluídos. Em amostragens de solo efetuadas em 3 épocas durante o ciclo da cultura, verificaram-se valores de umidade maiores para o nível $\mathrm{N}_{2}$. Segundo Rhoades (1972) o rendimento das culturas está relacionado à salinidade do solo integrada no tempo e se deve levar em consideração a concentração dos sais na solução do solo, que depende do teor de umidade.

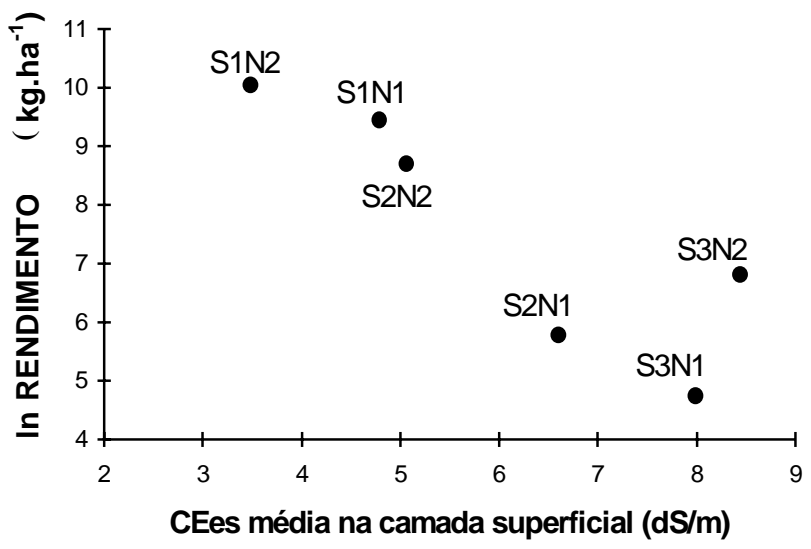

Figura 2. Rendimento do coentro em função da salinidade média do solo ao longo tempo, na camada de 0 a $10 \mathrm{~cm}$ e tratamentos estudados 


\section{CONCLUSÕES}

1. A altura e o número de hastes das plantas de coentro foram reduzidos com o aumento da salinidade da água e com diminuição da quantidade de água aplicada.

2. O stand final foi afetado drasticamente com o aumento da salinidade da água de irrigação, dependendo do nível de irrigação adotado.

3. Como conseqüência dos efeitos dos tratamentos na altura, no número de hastes e no stand final, a produtividade de folhas verdes resultante foi significativamente afetada pela salinidade e pela quantidade de água aplicada, que agiram interativamente na salinidade da solução do solo.

4. A produção comercial de coentro ocorreu apenas para os tratamentos $\mathrm{S}_{1} \mathrm{~N}_{1}, \mathrm{~S}_{1} \mathrm{~N}_{2}$ e $\mathrm{S}_{2} \mathrm{~N}_{1}$, cuja salinidade média do solo foi inferior a $5,1 \mathrm{dS} \cdot \mathrm{m}^{-1}$.

5. Há evidências de que o coentro, cultivar Arómatico, não consegue se desenvolver e produzir quando a salinidade do solo durante o cultivo fica acima de 7,0 dS.m ${ }^{-1}$.

\section{REFERÊNCIAS BIBLIOGRÁFICAS}

AYERS, R.S.; WESTCOT, D.W. A qualidade da água na agricultura. Campina Grande: UFPB, 1991. 218p. (FAO. Estudos de Irrigação e Drenagem, 29, rev. 1).

BRASIL. Ministério da Agricultura. Levantamento Exploratório: reconhecimento de solos do Estado do Rio Grande do Norte. s.l., DNPEA, SUDENE/USAID, 1971. (DPP. Boletim, 21PE13).

DOORENBOS, J.; PRUIT, W.O. Crop water requirements. Rome: FAO, 1977. 144p. (FAO: Irrigation and Drainage Paper, 24).

FERREIRA, P.V. Estatística experimental ligada à agronomia. Maceió: EDUFAL, 1991. 437p.

FILGUEIRA, F.A.R. Manual de olericultura: cultura e comercialização de hortaliças. São Paulo: Ceres, 1982. v.2, p.25-26.
FILGUEIRA, R.F. Efeito de diferentes níveis de salinidade do solo no comportamento de duas cultivares de coentro (Coriandrum sativum L.). Mossoró: ESAM, 1993. 46p. (Monografia de Especialização).

GOMES, F.P. Curso de estatística experimental. 12.ed. Piracicaba: Nobel, 1987. 430p.

LEPRUN, J.C. Primeira avaliação das águas superficiais do Nordeste: relatório de fím do convênio de manejo e conservação do solo do Nordeste brasileiro. Recife: SUDENE, 1983. p.91-141.

MAAS, E.V.; HOFFMAN, G.J. Crop salt tolerance - Current assessment. Journal of Irrigation and Drainage Division, v.103, n.IR2, p.115-134, June 1977.

MATIAS, V.D.P. Comercialização de hortaliças no Estado do Rio Grande do Norte. In: ENCONTRO DE HORTALIÇAS DA REGIÃO NORDESTE, 1, Mossoró, 1986. Anais... Mossoró: ESAM, 1986. p.40-51.

MEDEIROS, J.F. de. Qualidade da água de irrigação e evolução da salinidade nas propriedades assistidas pelo "GAT" nos Estados do RN, PB e CE. Campina Grande: UFPB, 1992. 173p. (Dissertação de Mestrado).

MEDEIROS, J.F. de; GHEYI, H.R. \& BATISTA, M.A.F. Procedimentos de análise de solo e água para diagnóstico de salinidade. Mossoró: ENA, ESAM, 1993a. 25p. (Coleção Mossoroense, Série E, 1256).

MEDEIROS, J.F.; GHEYI, H.R.; SILVA JÚNIOR, L.G.A. Efeito da salinidade da água de irrigação no desenvolvimento e produção do coentro (Coriandrum sativum L.). In: CONGRESSO BRASILEIRO DE ENGENHARIA AGRÍCOLA, 22, Ilhéus, 1993. Anais... Ilhéus: SBEA/ CEPLAC, 1993b. v.5, p.3141-3153.

RHOADES, J.D. Quality of water for irrigation. Soil Science, v.113, n.4, p.277-284, Apr. 1972.

RHOADES, J.D.; KANDIAH, A.; MASHALI, A.M. The use of saline waters for crop production. Rome: FAO, 1992. 133p. (FAO. Irrigation and Drainage Paper, 48).

SOUZA, A.F. Coentro (Coriandrum sativum L.). Brasília: EMBRAPA-CNPHortaliças, 1981. 5p. 